\title{
Complex application of instrumental analytical methods for detection and characteristics of polymer coating defects in drug-eluting stents
}

Background: The clinical performance of a drug-eluting coronary stent largely depends on the properties of its polymeric coating. While the randomized clinical research is the "gold standard" for the effectiveness and safety evaluation of the coronary stent, the bench top testing of the stent coating may provide valuable insights within the development of new devices. We argue that the combination of the instrumental analytical methods discovers more information of the nature and possible causes of irregularities of the stent coating than scanning electron microscopy conventionally used for this purpose.

Methods and findings: We examined bioresorbable coatings of three commercially available DES using scanning electron microscopy (SEM), scanning probe microscopy (SPM), nanoindentation, and X-ray photoelectron spectroscopy (XPS) to evaluate morphology (thickness in particular) and mechanical properties of the coating, as well as the of elemental and chemical composition through the coating thickness.

Conclusions: Adding of SPM and XPS methods to SEM for analysis of DES surface provides additional value, especially for the development of new polymeric coating. For the coatings being analyzed, the SEM method revealed the difference in the uncoated surface as big as $0.1 \%$ and $17 \%$ for different models of the stents. The data obtained by XPS method confirmed the results of the SEM-study about the surface uniformity. The depth profiling of the polymer drug-containing layers also showed the difference in thickness of the coatings as well as allowed to determine the elemental components of layers. The SPM method allows excluding the heterogenic or difference of elastic properties of the polymer coating as a cause for coating irregularity, that appear to differ only slightly for the stents with the smallest and largest uncoated areas.

Further research is needed to find the correlation of the properties discovered using the with analytical methods with characteristics of biological response.

Keywords: Drug-eluting stent" Biodegradable polymer" Coating defects" Scanning electron microscopy - Scanning probe microscopy - X-ray photoelectron spectroscopy= Stent surface

\section{Introduction}

Polymeric materials became widespread in implantable medical devices through processing capabilities in a wide range of variation in their physical and chemical properties as well as biocompatibility. Drug
Dmitry Kapustin*, Tatyana Dmitrieva², Alexander Rashkovskiy ${ }^{2}$ 'Shemyakin-Ovchinnikov Institute of Bioorganic Chemistry RAS, ul. Miklukho-Maklaya, 16/10, Moscow, 117997, Russian Federation ${ }^{2}$ Systems For Microscopy and Analysis, LLC

*Author for correspondence:

Tel.: +79162776094

Fax: +74953351011

kapustin@ibch.ru

Submitted: 28 March 2017

Accepted: 20 April 2017

Published online: 25 April 2017 
eluting polymeric coatings have substantially improved the clinical efficacy of coronary stents used to treat both chronic and acute conditions associated with a decrease in the lumen of the coronary arteries. The drug released in a few weeks or even months from the polymer coating prevents the inflammatory process and the associated re-narrowing of the coronary artery lumenrestenosis [1-5].

Randomized clinical research is the "gold standard" for the effectiveness and safety evaluation of coronary stent. Nevertheless, its implementation associates with the health risks for patients and significant costs. In addition, some complications are rare, and determination of their probability require large and lengthy clinical trials. In the short trials (which is often sufficient for product registration), these complications simply could not occur. In such case, the market obtain the product with "hidden problems" that increase risk of late adverse events that may happen when stents are already in widespread clinical use. On the other hand, there is a lack of published results of bench top testing of different types of drug-eluting stents (DES) provided by independent investigators. It should be noted that FDA recommends to complete all nonclinical tests of the finished DES before clinical trials and repeat it in any case of possible changes in DES during R\&D cycle (due to scale up of producing process from lab-scale for human tests to large manufacturing for commercial purpose) [6].

Polymeric coating must withstand all cycles of processing (crimping, sterilization and expansion) without compromising the integrity of the coating [7]. Therefore, on early stage of development of polymerdrug formulations and technologies of their application during the stent manufacturing, it would be beneficial to develop and apply methods of instrumental analytical control that can predict clinical efficacy and safety of coronary drug-eluting stents and anticipate possible risks of their use.

Aim of our study was to examine polymeric coatings of coronary stents not only with traditional optical and scanning electron microscopy, but also include such instrumental techniques as scanning probe microscopy, nano-indentation, and the method of X-ray photoelectron spectroscopy (XPS). Application of these instrumental analytical methods allows us to determine the chemical composition of the polymer coating, its thickness variations, and also to assess the morphological textural homogeneity and uniformity of distribution of the polymer coating on the stent surface in the crimped state and after balloon expansion. Such comprehensive approach enables to identify the possible causes of the irregularities of the polymer coating and provide feedback necessary for fulfilling the stent manufacturing technology for producing a batch production with reproducible quality indicators.

\section{Materials and Methods}

\section{Objects of research}

We investigated three second-generation DES with the biodegradable coating based on poly-lactic acid copolymer (Stent 1 DES, Stent 2 DES and Stent 3 BVS, four samples of each stent):

Stent 1: Endovascular metal stent with polymeric drug-eluting coating-Calipso (Angioline, Russia) before and after balloon expansion. Stent sizes: $3.0 \times$ $14 \mathrm{~mm}$. Coating composition: polymer-biodegradable copolymer of glycolic and lactic acids. Drug substanceSirolimus, labeled amount $-150 \mu \mathrm{g} / \mathrm{cm}^{2}$.

Stent 2: Endovascular metal stent with polymeric drug-eluting coating-Coracto (Alvimedica, Turkey) before and after balloon expansion. Stent sizes: $3.0 \times$ $24 \mathrm{~mm}$. Coating composition: polymer-biodegradable copolymer of glycolic and lactic acids. Drug substance - Rapamicine, labeled amount $-160 \mu \mathrm{g} / \mathrm{cm}^{2}$.

Stent 3: Endovascular polymeric stent with polymeric drug-eluting coating - Absorb (Abbott, USA) before and after balloon expansion. Stent sizes: $2.5 \times 12 \mathrm{~mm}$. Drug coating-biodegradable polymer (poly-D, L-lactic acid) and Everolimus, labeled amount-114 mg.

Totally, twenty seven DES samples were examined with microscopy methods:

- Three samples of each type DES for optical microscopy and SEM (before and after balloon expansion).

- Three samples of each type DES for XPS

- Three samples of each type DES for SPM

\section{DES preparing protocol}

The stents were unpacked immediately before each study with appropriate precautions to prevent contamination of the stent surface during manipulation. Stents were expanded according to manufacturer's flow chart to nominal diameter in air at $23-25^{\circ} \mathrm{C}$ (according to FDA Guidance [6]).

Investigation of morphology and texture of the surface of stents

The morphology of the surface layer of the polymer coating was investigated by scanning probe microscopy using Nano Indentation Tester NanoScan-3D 
(TISNCM, Russia); nanometer-range indentation was performed using the same device in accordance with the recommendations of the developer. Crimped stents were unpackaged, the protective plastic bag was removed, and the delivery system was cut off to fit the stent into the microscope chamber. A scanning of the surface was carried out in the mode of registration of the indenter vibration frequency changing. The area of the scanned surface was determined based on the geometric dimensions of stent struts claimed by the manufacturer (no more than $75 \mu \mathrm{m}$ in width). To ensure the fidelity of the results at least three scanning areas were selected proximal, central and distal ones. The obtained images were processed using NanoScan Viewer software. In the image processing the algorithms of subtracting of the surface curvature associated with the curvature radius of the stent design were used.

In parallel, the texture of surface layer of polymer coating was investigated by optical and scanning electron microscopy using a scanning electron-ion microscope Phenom Pro (Phenom-World B.V., Netherlands) and two-beam scanning electron microscope Versa 3D LowVac (FEI, USA). All DES were examined without additional metal layer spraying to keep the coating pure.

\section{Evaluation of the coating defects area}

An area of the stent coating defects was evaluated using the graphic editor Adobe Photoshop CC using the following procedure:

1. On the stent image the area of the stent structure was selected, excluding the zones related to the surface of the balloon and/or excesses of the polymer coating. An area of selected fields was calculated in pixels using the built-in analyzer software. The resulting value corresponds to the area of outer surface of the stent $\left(\mathrm{S}_{\text {outer }}\right)$.

2. Then, the contrasting zones of the stent structure were selected, which corresponded to the uncoated metal surface. An area of selected fields was calculated in the pixels using the built-in analyzer software. The resulting value corresponds to the area of uncoated surface of the stent $\left(\mathrm{S}_{\text {uncoated }}\right)$.

3. The proportion of uncoated area was calculated as percentage by the following formula:

$\mathrm{D}_{\text {uncoated }}(\%)=\left(\mathrm{S}_{\text {uncoated }} / \mathrm{S}_{\text {outer }}\right) \times 100 \%$

Evaluation of distribution of elemental and chemical composition through the coating thickness

Profile distribution characteristics of elemental and chemical composition of the coating from the article

\begin{tabular}{|l|c|}
\hline \multicolumn{2}{|c|}{ Table 1: XPS investigation parameters. } \\
\hline The energy of primary X-rays & $1486.7 \mathrm{eV}$ \\
\hline The spot diameter of primary X-ray beam & $10-50 \mu \mathrm{m}$ \\
\hline Transmission energy of electron analyzer, eV & $187.85 ; 46.9 ; 23.5$ \\
\hline The angle of collection of secondary electrons & $45^{\circ}$ \\
\hline Exposure time & $20-50 \mathrm{~ms} / \mathrm{channel}$ \\
\hline The size of the energy channel & $0.8 \mathrm{eV}, 0.1 \mathrm{eV}$ \\
\hline Etching of the surface with argon ions & $\mathrm{Applied}$ \\
\hline The energy of the primary ion beam & $3 \mathrm{keV}, 5 \mathrm{keV}$ \\
\hline The area size of etching with argon ions & $2 \times 2 \mathrm{~mm}$ \\
\hline Scanning type in the analysis at the point & Point, Area \\
\hline Etching step in depth & $200 \mathrm{~nm}$ \\
\hline The size of field in the analysis at the point & $1.2 \times 1.2 \mathrm{~mm}$ \\
\hline Neutralization of charge & $\mathrm{Applied}$ \\
\hline
\end{tabular}

surface to a depth of 6.5-6.7 $\mu \mathrm{m}$ was obtained by X-ray photoelectron spectroscopy using X-ray photoelectron spectrometer PHI VersaProbe II 5500 (ULVAC-PHI Inc., USA) with the parameters listed in Table 1.

The elemental composition of the coating with a thickness less $10 \mu \mathrm{m}$ was determined with depth intervals of $200 \mathrm{~nm}$.

The samples of stents were placed on the stage with a diameter of $25 \mathrm{~mm}$. Then the points for analysis were selected. The image was registered in secondary electrons to select the zones for collection of XPS spectral data at depth profiling. XPS spectra were recorded on each sample surface at selected points (they are identified on the corresponding figures in the sections "Results" and "Discussion").

Depth profiling of the samples was consisted of XPS spectra registration at selected points during the successive removing of equal layers of coating material from the sample surface by the etching with ions $\mathrm{Ar}^{+}$.

The obtained data were used to calculate the concentrations of all elements detected in the composition of the coating layer and the distribution profiles were plotted in accordance with the following procedure.

\section{Method for calculating of XPS parameters}

The measurement parameters are listed in Table 2 . The elemental composition of the coating was determined with depth intervals of $200 \mathrm{~nm}$ through the depth at different points on the surface of samples by analysis of the obtained XPS spectra using MultiPak 9.0 software. To determine the number and relative concentration of different types of bonds involving the detected chemical elements, the particular XPS spectra of the most intensive spectral lines of elements contained in the coating were analyzed using the iterative matching 


\begin{tabular}{|l|c|}
\hline Table 2: Parameters used in the measurements. & Value \\
\hline \begin{tabular}{l|c|} 
Parameter & MultiPak 9.0 \\
\hline Software & Applied \\
\hline Curve Fitting procedure for individual spectral bands & $1.0-2.0 \mathrm{eV}$ \\
\hline The full width of the spectral line at Half Maximum (FWHM) during the Curve Fitting procedure & Applied, for line C1s \\
\hline Correction of surface charge & Not less than 20 \\
\hline The amount of the statistical sample to measure a single parameter & \\
\hline
\end{tabular}
\end{tabular}

procedure "Curve Fitting". The type of the formed bond was determined from the chemical shift (position on the energy scale) of the detected components of spectral lines of the known compounds.

\section{Results}

\section{Optical and scanning electron microscopy}

The two types of SEM were used. Rapid evaluation was carried out using a desktop device PhenomPro with a detector of back-scattered electrons, providing a quickly assessment of coating at the macro level. Detailed study of the coating topography was perfomed using a scanning microscope Versa 3D with a detector of secondary electrons. Crimped stent was placed in a chamber of microscope, and the image was taken. Then, the stent was expanded according to the manufacturer's recommendations and the next image was taken without removing the balloon. As a result, the information about changing of the coating morphology during the stent expansion was obtained, and adhesive strength of the coating was qualitatively estimated. The results are presented on Figures $1-12$.

\section{X-ray photoelectron spectroscopy}

XPS was used to determine the elemental composition of the coating. It allowed us to investigate the elemental distribution and to evaluate uniformity of the polymer layers as across the surface of stents (mapping) as well as composition of polymer drug-containing layer through the depth. The results are presented on Figures 13, 14.

\section{Scanning Probe Microscopy}

The surface morphology of polymer layer was studied in detail with the identification of the nature of defects at the micro level, and secondly, the distribution of the elasticity modulus of studied coatings was obtained. The obtained $2 \mathrm{D}$ and $3 \mathrm{D}$-images of the surface in combination with the corresponding profiles of the coating thickness changing in an arbitrary direction, as well as analysis of these profiles allowed us to make a conclusion about the uniformity of a coating distribution on the substrate surface and about the medium size of coating defects. Figure 15 shows 2D and 3D-images of the surface areas of biodegradable coating of Stent 1 and the related profiles. Other results of the study of the surface morphology and the module of elasticity of Stent 2 and Stent 3 polymer coating by SPM method are shown in Figures 16 - 20.

\section{Discussion}

The published results of comparative studies of clinical efficacy relating to the properties of stents with polymer coatings do not consider the physical-mechanical and morphological properties of the coatings themselves. However, these characteristics of the coating may affect the clinical outcomes after application of the stent. Thus, the low adhesion properties and crispness of coating may cause the entering of polymer particles into the bloodstream and increase the risk of embolism, microvascular obstruction of vessel and/or myocardial necrosis. Deformations of integrity and uniformity of the coating may reduce the therapeutic effect of the drug and increase the risk of stent thrombosis. Therefore, the results of evaluation of biological response (due to in vitro, in vivo and clinical studies) may be affected by both the initial physical and mechanical properties of the coating, and their changes in the course of the stent life cycle.

At the first stage in this work, a comparative study of properties and morphology of the biodegradable coatings of stents was carried out. To describe the discovered irregularities we used the classification of irregularities developed by Basalus et al. [8]. This classification, which includes four categories, is based on the evaluation of two basic characteristics: the thickness of the polymer coating and the degree of coating displacement (or delamination).

Category I. Irregularities with reduced thickness of coating.

IA Small or big areas with aspect of bare metal.

IB Cracks.

IC Reduced thickness of stent coating at strut crossings.

Category II. Irregularities with increased thickness of coating.

IIA 'Auricle-shaped' excess of coating. 
IIB Ridge-shaped excess of coating connecting two facets of a strut.

IIC Small rounded structure of excess coating.

IID Coarse irregular excess of coating.

Category III. Irregularities with inhomogeneous thickness of coating.

IIIA Crater-shaped irregularity with metal exposure.

IIIB Crater-shaped irregularity without metal exposure.

IIIC Small crater-shaped irregularity with appearance of punched-out hole.

IIID Wrinkles.

IIIE Flattened coating enclosed between two linear thickenings of coating material.

Category IV. Irregularities with displacement of coating.

IVA Webbing with metal exposure.

IVB Webbing without metal exposure.

IVC Fragments of coating.

IVD Torn webbing.

IVE Peeling of polymer without bare metal exposure.

IVF Peeling of polymer with bare metal exposure.

The irregularities of polymer coatings discovered in this study were attributed to the relevant paragraphs of this classification.

Figure 1 shows the images of surface of stents coated with polymer compositions, obtained by optical microscopy. Visible irregularities of stent coating are not observed at magnification (40x). However, at magnification $80 \mathrm{x}$ nodules and irregularities of biodegradable coating (defects IIC and IID) are visible. Thus, the presence of coarse defects of biodegradable coating can be determined by optical microscopy as late as relatively low magnification (80x).

A more detailed study of elements of the stent coating surface is provided by SEM method. Analysis of the images makes it possible not only to classify the individual defects, but also to make assumptions about the reasons for their occurrence. It was turned out after an analysis of images and calculation of the area of uncoated sites using the graphical editor Photoshop CC (Adobe), that for the stent Stent 1 this characteristic reached $17 \%$ of the surface area of the whole stent structure. A substantial share of the uncoated areas accounts for the side walls of struts.

Figure $2 \mathrm{~B}$ represents the region containing the coating exfoliation (Cat. IVE) and the dried pool (cat. IVC) of polymeric material which is formed, probably, as a result of excessive amount of polymer solution application. Apparently, it is a consequence of applying of the polymer composition by dipping of the stent into the polymer solution or melting. The presence of such pools, obviously, poses a threat of the exfoliation of the material fragments of up to $100 \mu \mathrm{m}$ and their penetration into the bloodstream during the installation of the stent, which increases the risk of embolism.

Without accurate information about the conditions of the polymer coating formation, which is protected by the manufacturer, it can be assumed, however, that the stent is secured on the catheter immediately after application of the polymeric composition, prior to the step of composition drying/curing. Thus, the formation of the coating on the stent surface was carried out when the stent was already on a balloon. This conclusion is confirmed by the balloon images shown in Figure 3 . The imprints of stent material on the surface of the balloon after the stent removal are well defined.

Investigation of another sample of DES with a polylactic coating-Stent 2 (Coracto, Alvimedica) shows that the producer is able to obtain uniformly

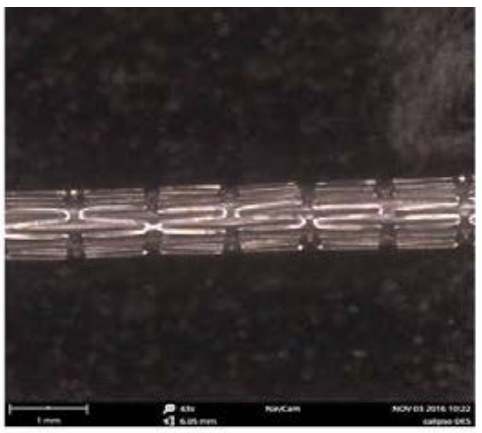

A

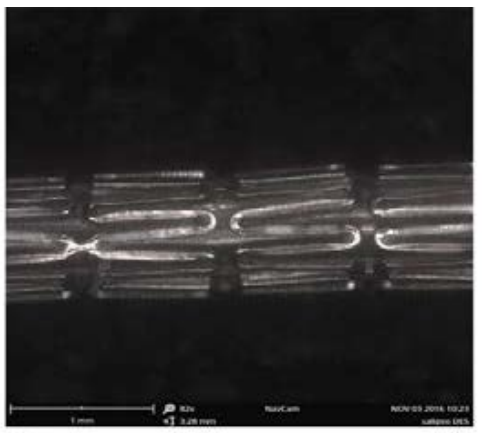

B

Figure 1: Images of the Stent1 mounted on the delivery system obtained by optical microscopy with PhenomPro device. 


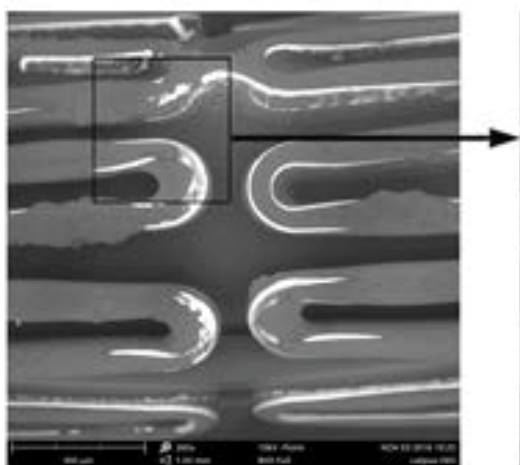

A

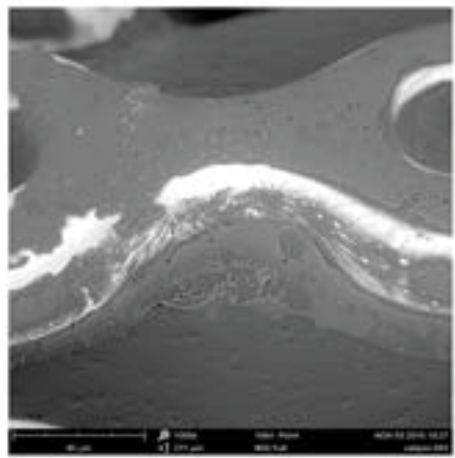

C

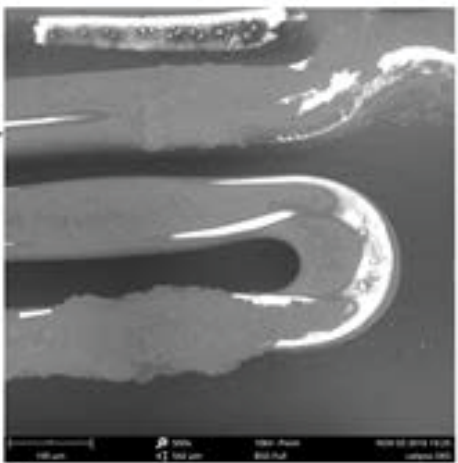

B

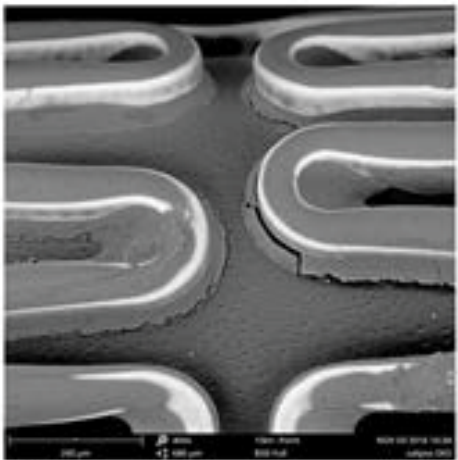

D

Figure 2: Surface images of the same Stent 1 (Figure 1) obtained with Phenom-Pro device in SEM-mode. (A) Strut surface at 265x magnification; (B) Dedicated site at 500x magnification; (C) The site of struts connection at 1000x magnification; (D) Section with 540x magnification.
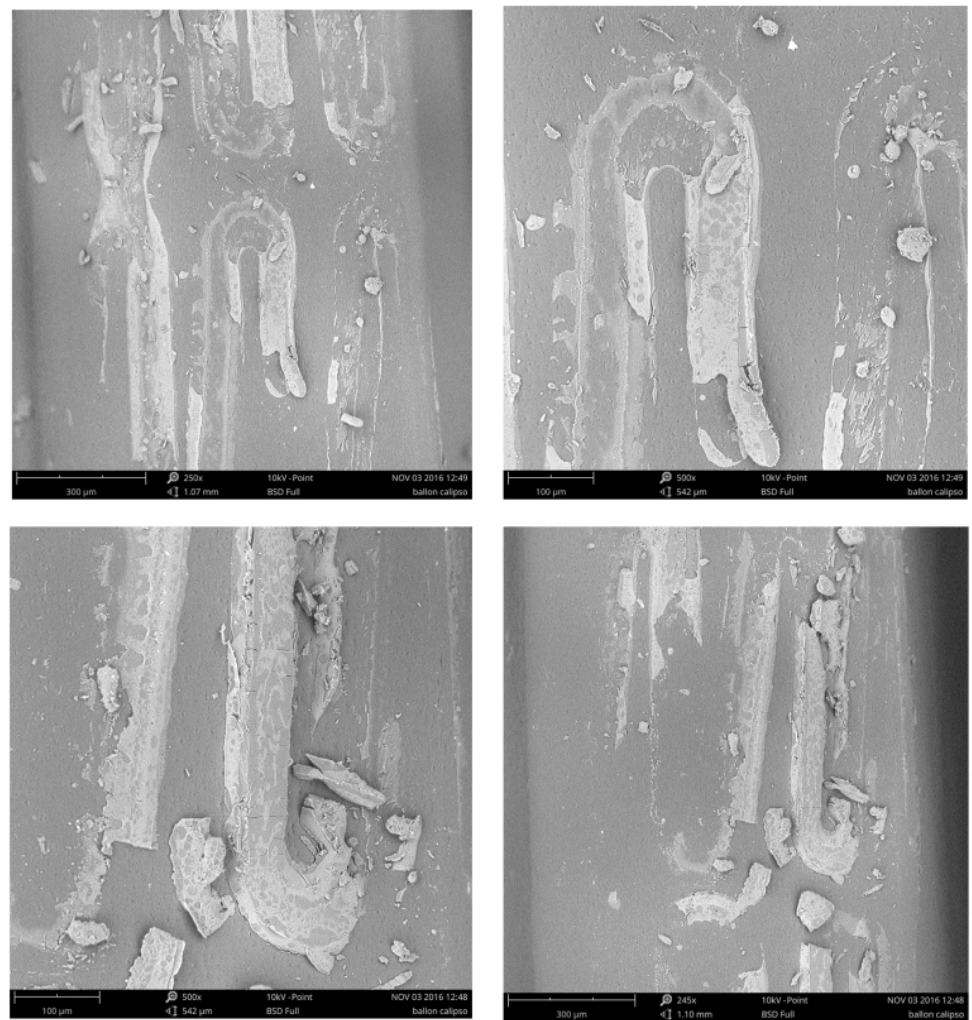

Figure 3: The imprinting on the balloon repeating the shape of the stent after its removing from the balloon surface. 
distributed and morphologically uniform polymeric coating (Figure 4). Share of uncoated sites according to the graphical analysis is about $0.1 \%$ for the crimped stent. Presumably, the evenness of the coating is achieved by spraying of polymer layer by layer, so the coating does not form drips or agglomerates in the corner sites. However, one can see that the coating has a number of defects resulting from subsequent crimping, such as cracks (Cat. IB), thinning of the coating at the intersections of struts (Cat. IC) and areas of delamination of less than $50 \mu \mathrm{m}$ (Cat. IA) at the points where the struts are contacted. Afterwards this may cause a local delamination of the polymer layer during the stent expanding (it is worth noting that visible defects can be absent in the case of satisfactory mechanical and elastic properties of the polymer coating).

The high quality of the coating deposition is confirmed by SEM images of the balloon after expanding and removal of the stent (Figure 5). Only light outlines of the stent structure are visible on the images, which are formed, presumably due to crimping of the material of balloon.

Detailed study using high resolution SEM (Versa 3D) shows defects both on the outer and on the inner

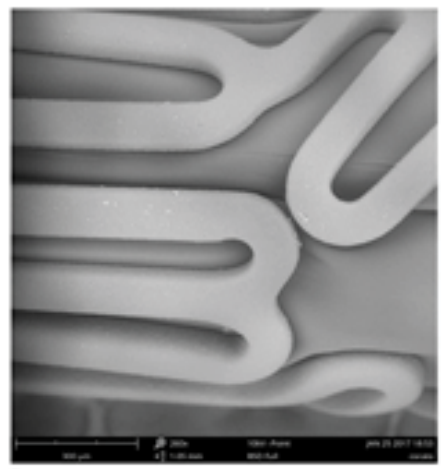

A

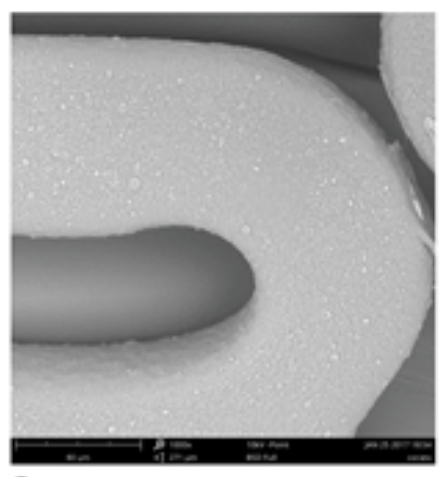

C surface of the stent-drips of biodegradable coating (Cat. IIB and IID), flaking of the coating (Cat. IVE, IVF), thickening of the coating (Cat. IIA) (Figures 6-8). Apparently, after application of polymeric composition the inner surface of stent was pressed against the surface of the balloon (and not only to the holder, since in this case the plate areas of the surface could be observed at small local sites). As a result, in the process of curing the coating was formed with the formation of areas with different topology (breaks and sinters are clearly visible in the convex fields; the light-colored thinner layer corresponds to the points of contact with the balloon surface in the plate areas). Flat surface and cracks caused by separation from the surface of balloon, as well as webbings and their breaks in the strut corners are clearly visible in Figures 4-6, respectively (Cat. IA, IB, IVA, IVB, IVD).

In the same manner, we examined the Stent 2 with a polylactide polymer coating before and after expanding. In the following images at low magnification, the bright and dark areas on the surface of the stent can be seen (Figures 9A-9C). The presence of such areas indicates a nonuniformity of the coating thickness while its continuity is maintained. Uncoated fields and drips of polymer composition in the points of contacts of struts with a balloon have not been identified.

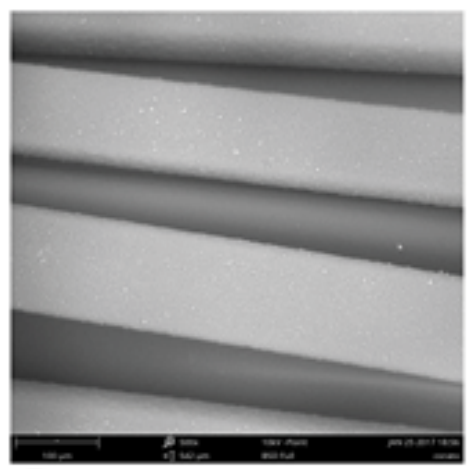

B

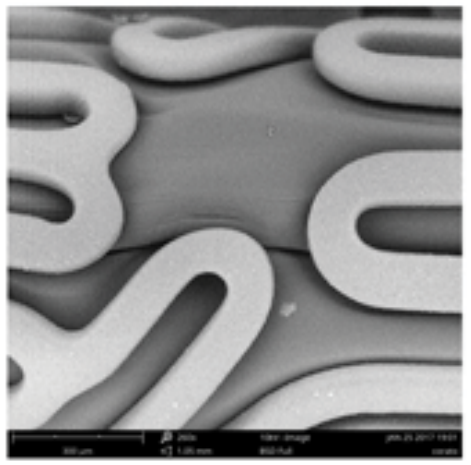

D

Figure 4: A series of images of the stent Stent 2 mounted on the balloon. (A-D) Different sites at various magnifications (from 260x up to $1000 \mathrm{x})$. 
After expanding, the thinning of the layer is observed: the share of light-colored fields in Figure $10 \mathrm{~A}$ is increased, but the coating integrity is generally maintained. Defects (Cat. IIIA and IIIB) are found only in the tops of adjacent struts in the form of bare sites of an oval shape, which are formed after expanding, presumably due to the contacts between struts during the coating formation (Figures 10C and 10D).

To determine the causes of the origin of the biodegradable coating defects in more details, we examined other model of drug-eluting stents-a fully

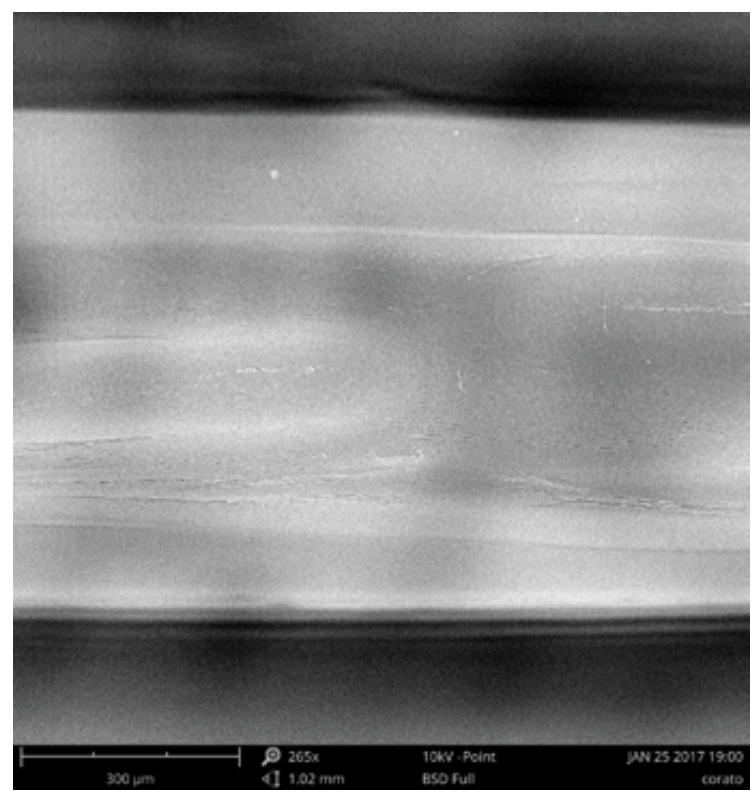

Figure 5: SEM images of the balloon after removal of the Stent 2.

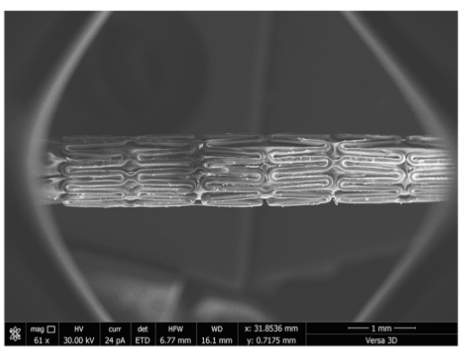

A

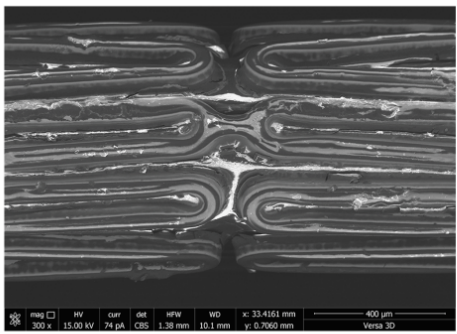

C biodegradable Absorb Abbott stent (Stent 3). BVS (bioresorbable vascular scaffold) is the late-generation of coronary stents are becoming a promising in treatment of young patients due to low damage of the treated vessel [9]. This stent was used as a sample for comparison, which is made on the basis of polylactic acid, that initially determines the high adhesion of the polymer coating to the base of the stent.

A detailed study of the Stent 3 surface in crimped state by SEM showed no major defects (Figure 11) related to the violation of the coating integrity, or in adhesion of the coating to the surface of the stent (Cat. IV). But it is worth noting the presence of areas on the inner surface of strut corners, where the folding of the surface is clearly manifested at $1000 \mathrm{x}$ magnification (Cat. IIID). In addition, the outer surface of the struts in the points of bends is characterized by the presence of material projections over of the total surface of the stent. In these areas undulating variation of the coating thickness appears, follows the bend of the strut. After expanding Stent 3 we could observe the cracks (Cat. IB) in outer sides of the struts, that could be caused by damage of the construction of the stent (Figure 12).

Compared with the SEM method, the XPS method does not provide such wide possibilities for visualization of various defects of the coating surface, but this method allows one to obtain the information about the thickness and uniformity of the coating and, more importantly, to evaluate the chemical composition of the coating throughout its depth. Thus, it is possible to obtain information about the homogeneity of the

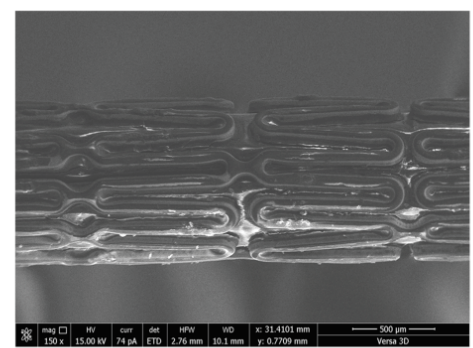

B

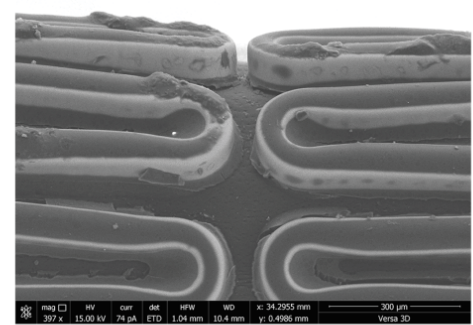

D

Figure 6: Images of surface of crimped Angioline Calipso (Stent 1) obtained using SEM with secondary electron detector. The lighter areas on the surface of the stent indicate the uncovered sites. 

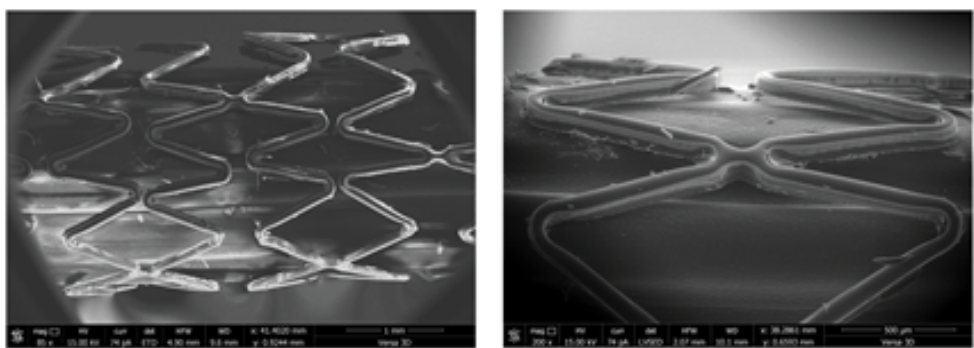

A

B
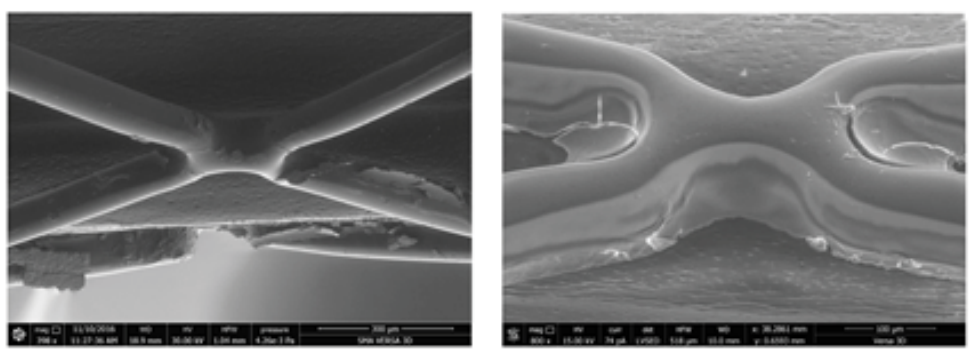

C

D

Figure 7: The same sample (see. Figure 6) after its expanding. Cracks, delamination of the coating material are clearly visible.

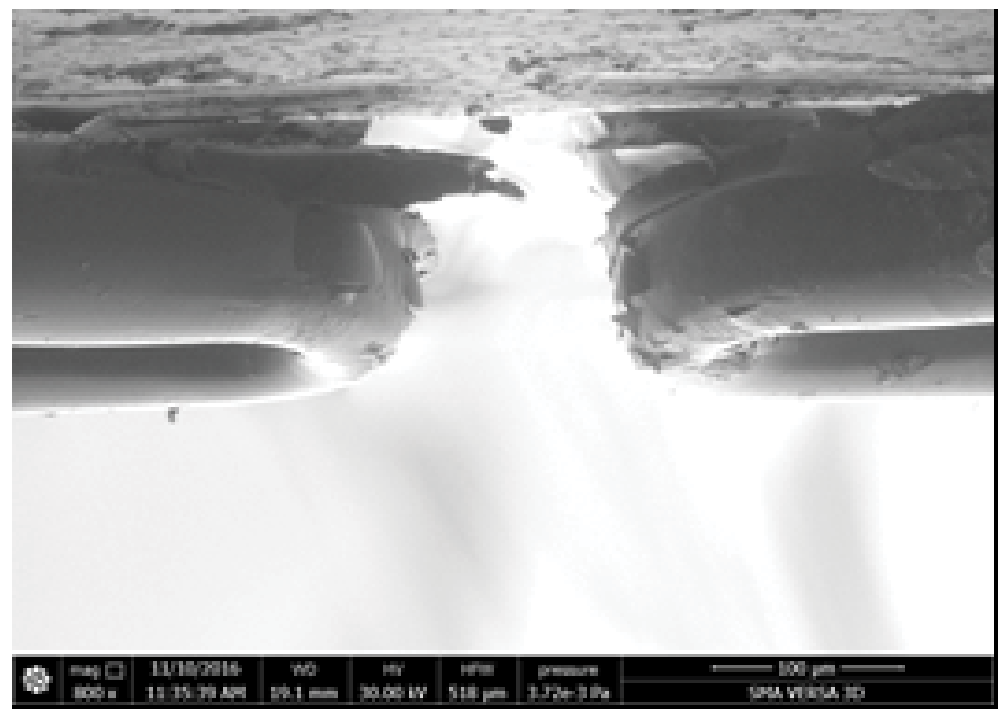

Figure 8: The flat inner surface with defects caused by separation of the coating sinter from the balloon during expanding.

coating material, the presence of layers of different compositions (the presence of an additional adhesive layer, multilayer coverage, etc.), and on the distribution of additional agents (drugs, radiopaque agents, etc.).

In Figure 13 showed the so-called XPS-map for the two stent samples studied. The color intensity in the images is proportional to the number of secondary electrons. The maximum intensity corresponds to the largest number of secondary electrons detected from areas with a thinner polymer coating. The obtained data confirm the results of the SEM-study of the nonuniformity of the polymer coating in a case of Angioline stent and variable thickness in Coracto case.
The depth profiling of the coatings on a $50 \mu \mathrm{m}$ area also showed the heterogeneity of the polymer coating of Stent 1. In The Figure 14 presents the profiles of changes in the concentration of elements to a depth of 6 microns. The presence of carbon (C1s) and oxygen (O1s) is determined by the content of organic components - polymer coating and drug agent, the presence of nitrogen (N1s) is determined by the content of the drug substance (sirolimus), the presence of metal atoms is determined by the composition of the metal substrate.

The data presented in Figures 13 and 14 show that in the case of a stent 1 coating the simultaneous presence 


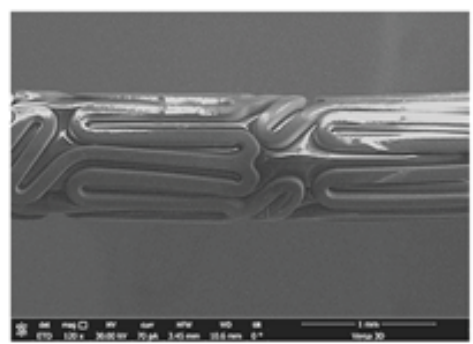

A

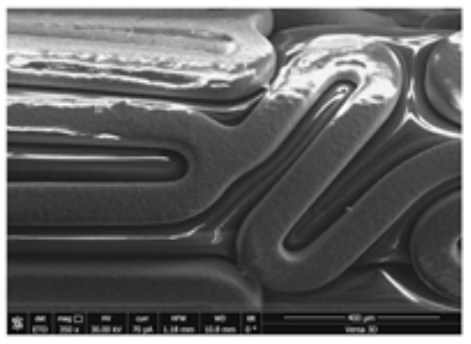

C

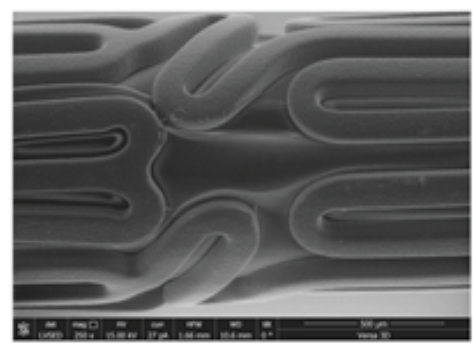

B

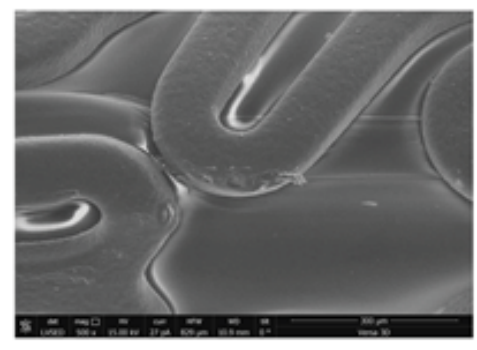

D

Figure 9: Images of surface of crimped Alvimedica Coracto (Stent 2) obtained using SEM with secondary electron detector.
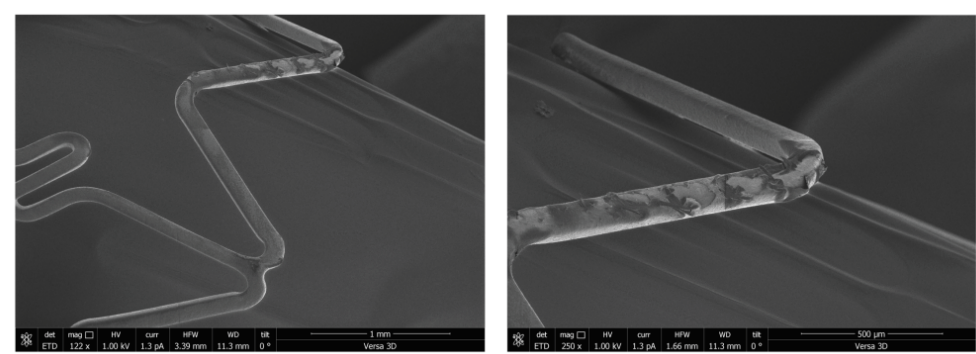

A

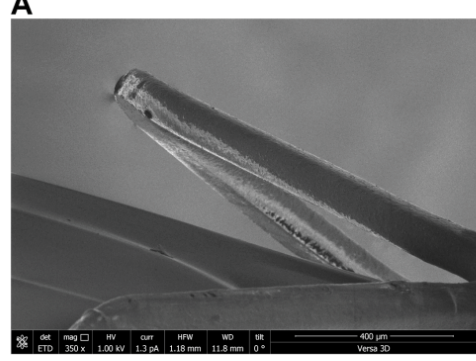

C
B

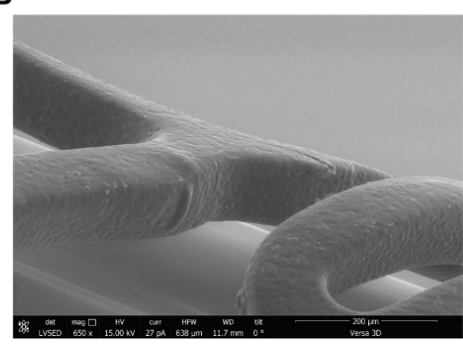

D

Figure 10: The Stent 2 (see Figure 9) after its expansion.

of carbon signals (from polymer component) and metal ones is observed even at a depth of $2.5 \mu \mathrm{m}$. Therefore, the average effective coating thickness is about $2.5 \mu \mathrm{m}$. Taking into account the XPS profiling data, it can be concluded that such a low value is determined by defects of the coating uniformity. Distribution of the drug (the characteristic signal of oxygen) in the content of polymer composition is relatively uniform throughout the etching depth. Obviously, the drug release profile will be determined by the rate (and the mechanism) of polymer biodegradation.
The effective thickness of a Stent 2 polymer coating was on average $4.5 \mu \mathrm{m}$. Apparently, an adhesive was added to the polymeric composition (it is indicated by the $\mathrm{Cl}$-signal that increase through the depth and reaches the maximum value near the metal substrate). The drug in a coating is distributed in the upper layers that correlates with a lot of crystallites observed on SEM images.

Thus, the combined use of SEM and XPS methods allowed us to elicit the key differences between 


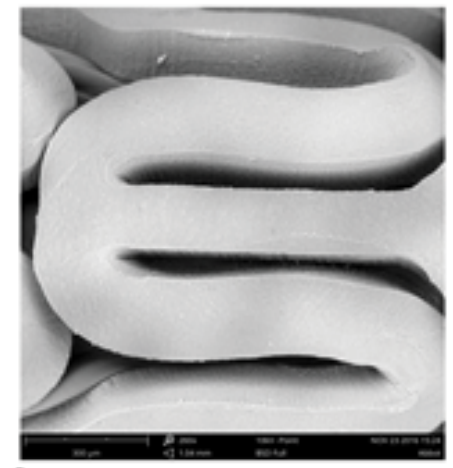

A

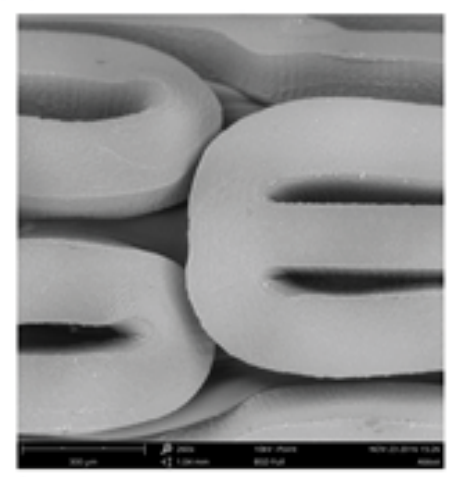

C

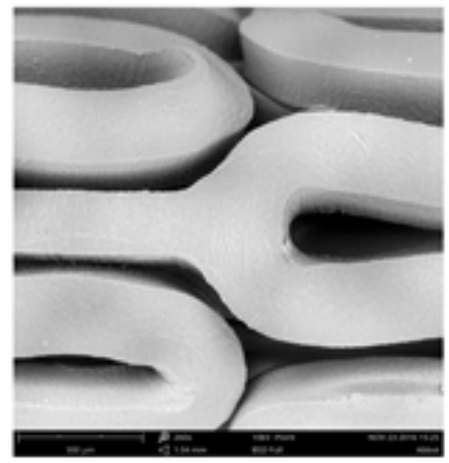

B

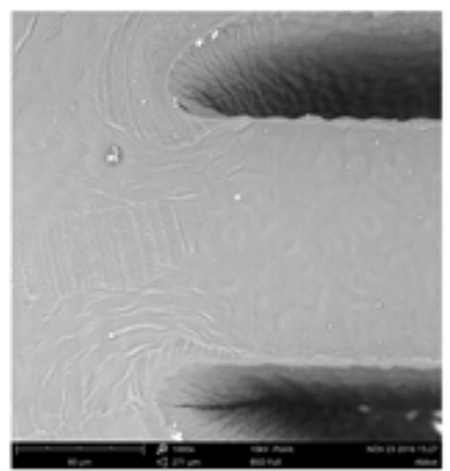

D

Figure 11: A series of images of the Abbott Absorb BVS (Stent 3) obtained using SEM via PhenomPro.

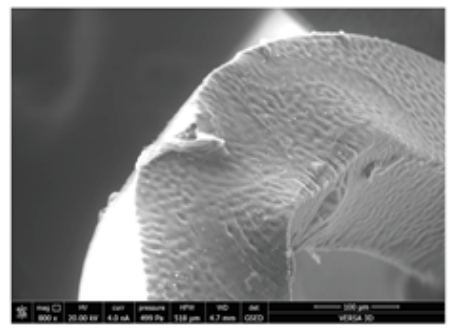

A

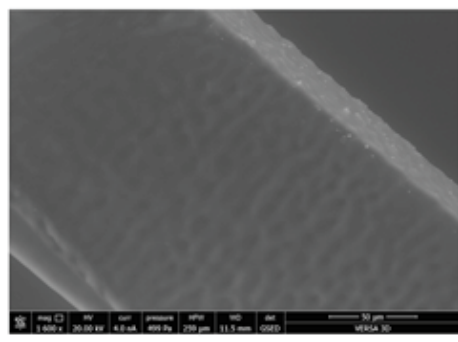

C

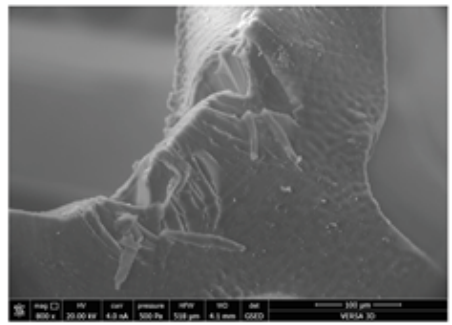

B

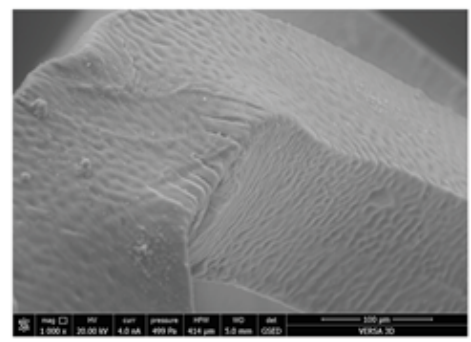

D

Figure 12: The coating and stent body irregularities of Stent 3 after expansion.

morphological characteristics of polymer coatings. In both cases, there is uneven thickness of the formed layer, but the coating of Stent 2 meets FDA requirements for integrity, unlike the Stent 1 coating. In addition, a Stent
1 coating has poor adhesion to the surface of metal alloy, how it is indicated by the presence of almost completely uncoated vertical sections of struts and delamination of the coating after the stent expanding. 


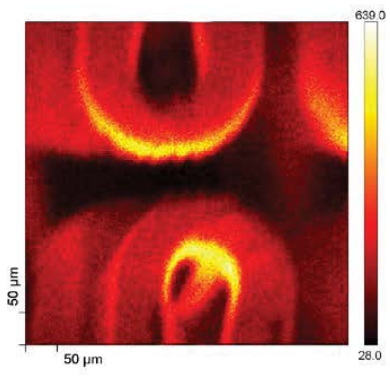

A
B

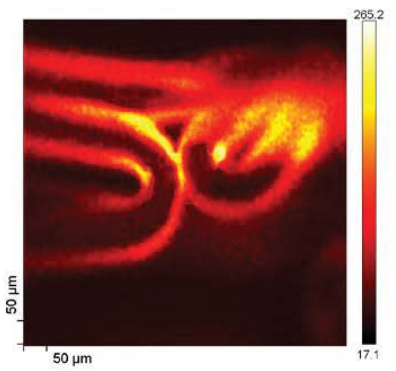

Figure 13: XPS cards of sites of the Stent 1 and Stent 2.

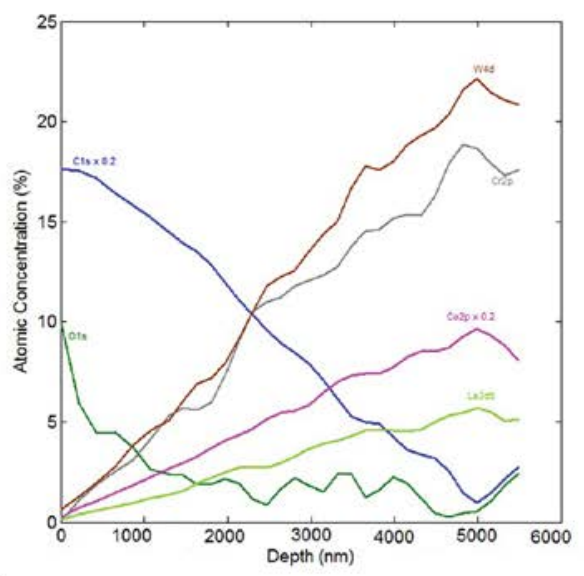

A

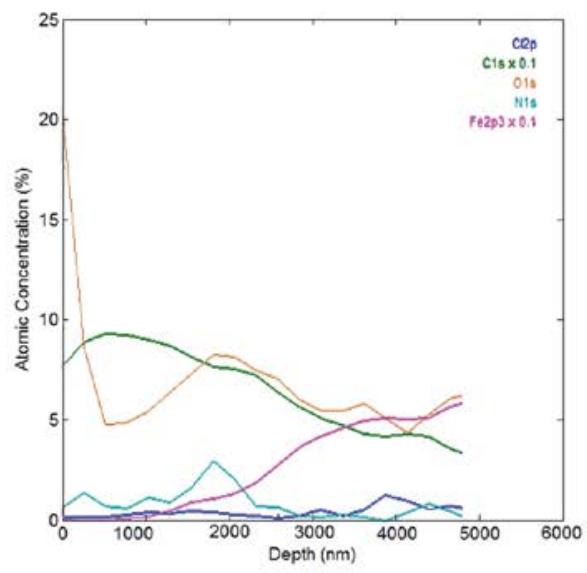

B

Figure 14: Examples of the elements concentration profiles defined in the upper layers $(0-7 \mu \mathrm{m})$ for Stent 1 and Stent 2.

The causes resulting in appearance of defects in the polymeric coating during its formation on the support surface may include unsatisfactory physical and mechanical properties of the coating and insufficiently developed technology of the coating deposition, which does not provide a uniform distribution of the polymeric composition over the substrate surface.

For detailed study of possible causes of irregularities we used the Scanning Probe Microsocopy (SPM) method.

All the images in Figure 15 show defects that could result from the stent crimping before enough drying of the coating or due to drying conditions that do not provide uniform and complete removal of the solvent. This observation is confirmed by the presence of split bubbles of about $10 \mu \mathrm{m}$ in diameter (Cat. IIIB) in some sites of the surface near the folds and laminations consisting of a polymeric material.

It can be assumed comparing the SEM images of the stent mounted on the balloon, and the results of SPM, that the stent after the coating formation and crimping was further subjected to thermal treatment. This is evidenced by smooth uncoated stent sections, especially in the edge and corner areas, as well as the defects identified in the site 1 (Figure 15) the nodules of irregularly shape (Cat. IIA), as well as solidificated relief of the surface reflecting the drift of not yet solidified material in a certain direction. Absence of the ordered structure in the coating relief demonstrates the use of mixed type or amorphous polymer component.

The measurement results of supply curves to obtain the primary data on the mechanical properties of the coating showed that the coating is characterized by a rather high elastic modulus of about $64.47 \pm 4.79$ (7.42 \%) GPa. At the same time the mechanical properties distribution map (prepared according to the angle of supply curve inclination) shown in Figure 16 demonstrates the relative uniformity of the coating material regarding to the modulus of elasticity.

Results of the study of the surface morphology and the module of elasticity of a Stent 2 polymer coating by SPM method are shown in Figures 17 and 18.

Typical layered clusters in the pattern of relief of the coating indicate the high crystallinity of the polymer used in this type of coating. The molecular chains in 

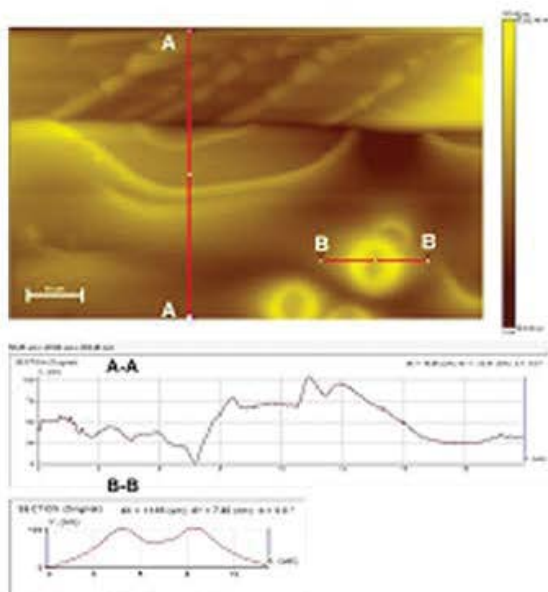

A

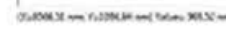

B

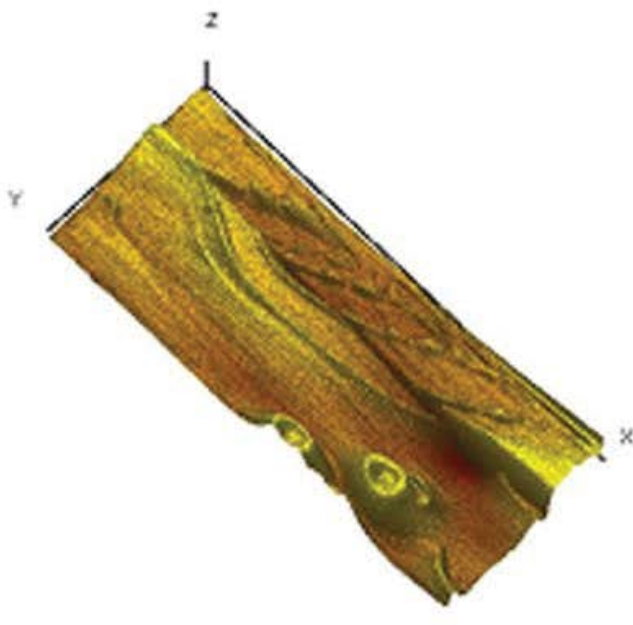

Figure 15: Examples of 2D and 3D-images of areas of Stent 1 surface coating and the corresponding reliefs obtained by SPM. The area size $50.28 \mu \mathrm{m} \times 20.04 \mu \mathrm{m}$.

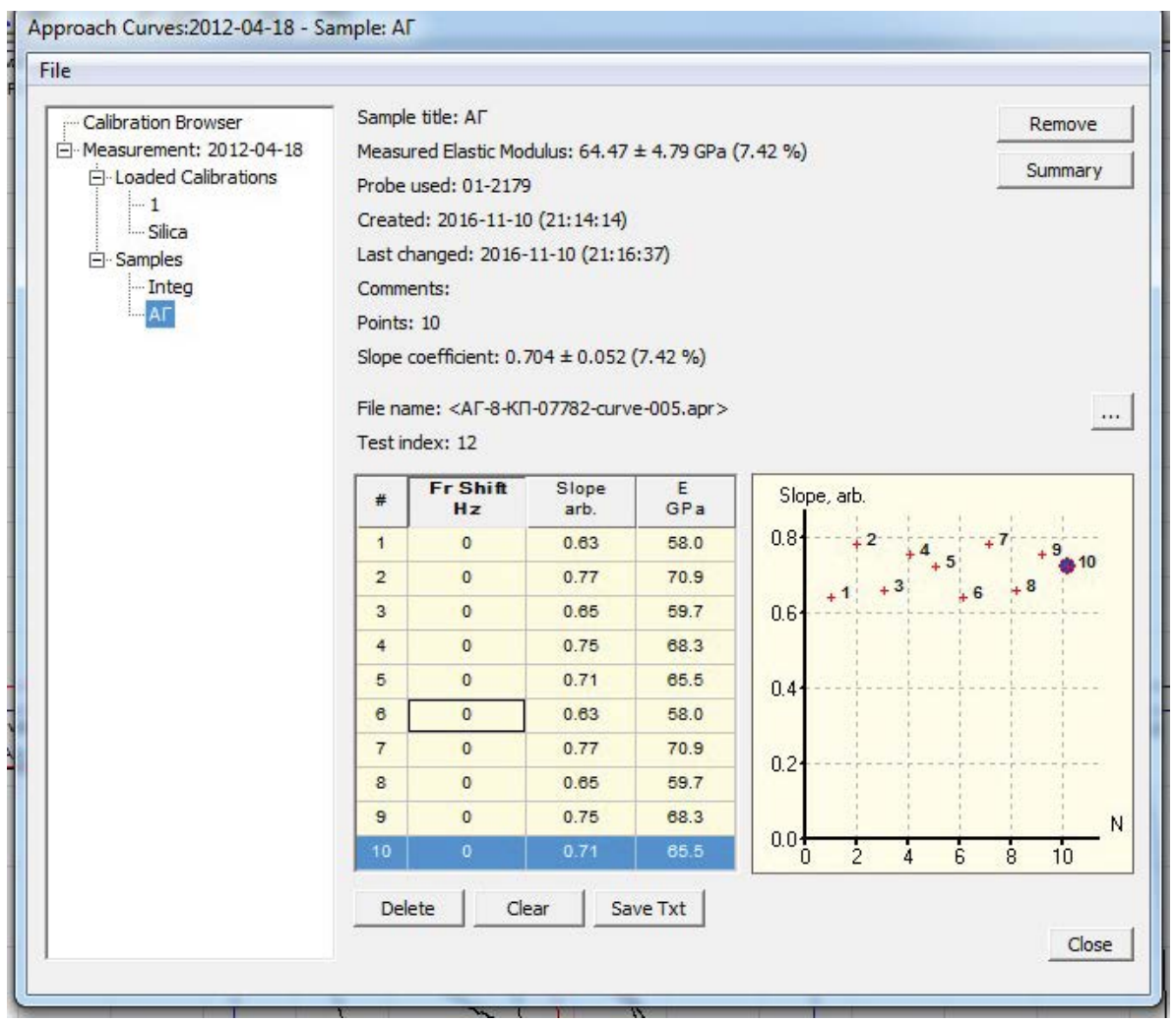

Figure 16: The results of the plotting of supply curves for the Stent 2.

the crystalline polymers have a folded conformation, which is characterized by the presence of recurring bends (folds) of the straightened segments of the polymer chains, equally spaced in parallel to each other. The results of calculation of the elastic modulus on the supply curves and a map of the coating mechanical properties distribution are shown in Figure 18. The mean value of the modulus of elasticity was $53.88 \pm$ 4.57 (8.47\%) GPa.
The images in Figure 19 show the parts of strut of the Stent 3. Typical layered clusters in the coating relief indicate the presence of sites in the polymer coating with a crystal structure (preferably, they are formed on the sidewalls of struts). The coating on the central sites of the struts has significantly more flattened, that confirms the presence of amorphous component in the polymer coating. Such microphase separation in the structure of material may indicate about the use 
of copolymer of lactic acid with glycolide as a polymer base of a coating or about the use of polylactic acid with a low molecular weight $(10-20 \mathrm{kDa})$. The presence of amorphous sites in the latter case is likely determined by the presence of water molecules adsorbed by polymer macromolecules. A more detailed study of the coating composition is possible using additional physical and chemical methods of analysis.

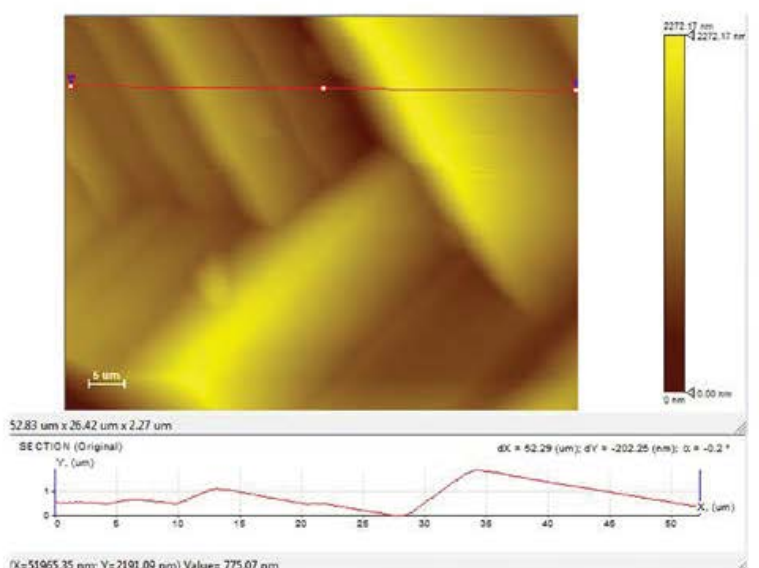

A

Figure 17: 2D and 3D-images of areas of the Stent 2 surface and the corresponding reliefs obtained by SPM. Field size $52.83 \mu \mathrm{m} \times$ $26.42 \mu \mathrm{m}$.
The mean value of the modulus of elasticity for Stent was $17.73 \pm 1.54(8.70 \%)$ GPa. The mechanical properties distribution map (obtained according to the angle of supply curve inclination) shown in Figure 20 demonstrates the relative uniformity of the coating material regarding to the modulus of elasticity.

Thus, a comparative analysis of the three polymer

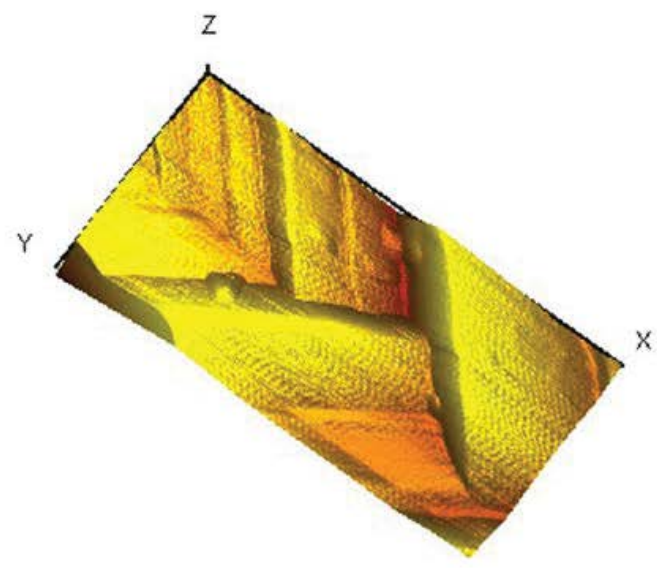

B

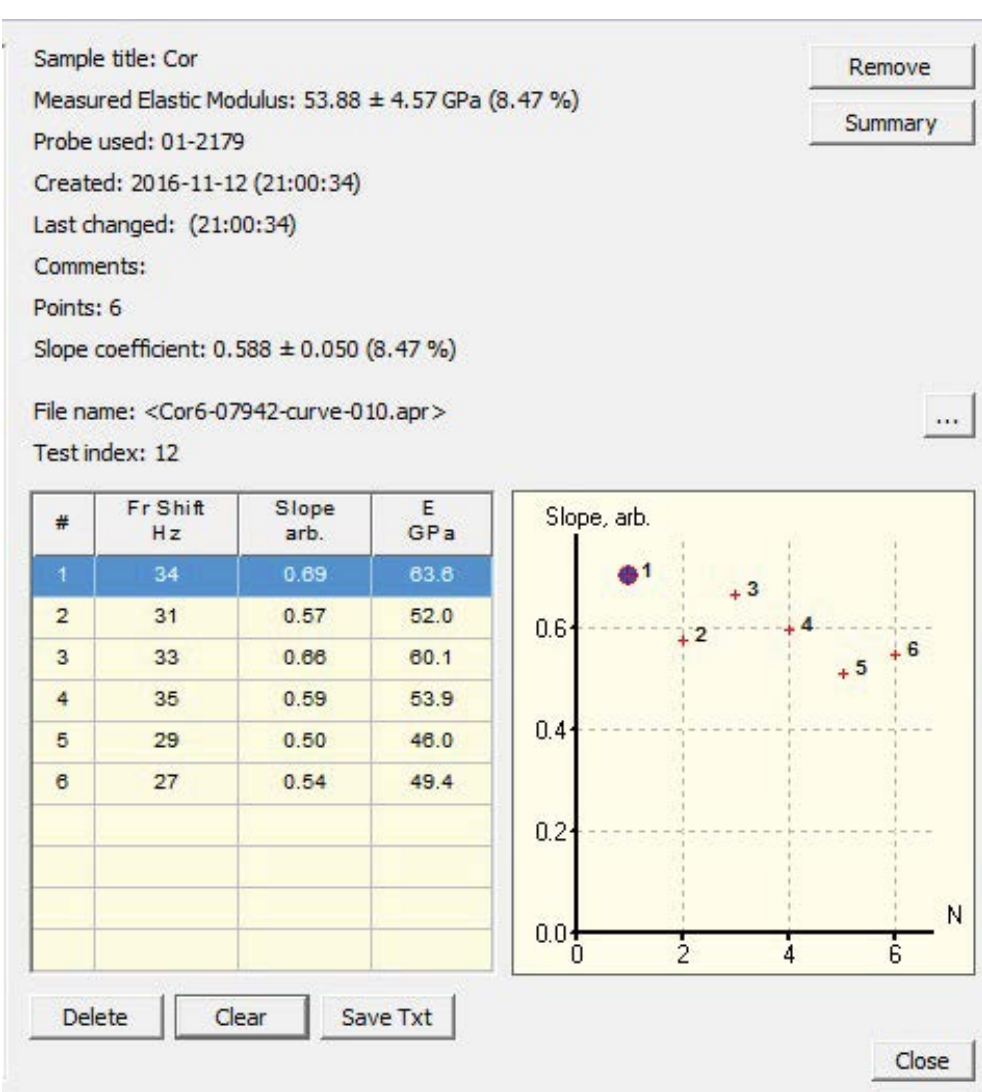

Figure 18: The results of the plotting of supply curves for the Stent 2. 
coatings using various microscopy techniques shown that a biodegradable coatings could have virtually all types of typical defects, where predominant categories are associated with the non-uniform deposition of the polymer composition on the main stent construction surface. The above described defects of a coating can be firstly connected with the low adhesion of the polymer composition to the metal surface (or due to the absence of an intermediate adhesive layer, the presence of which was identified in the study of a Stent 2 polymer

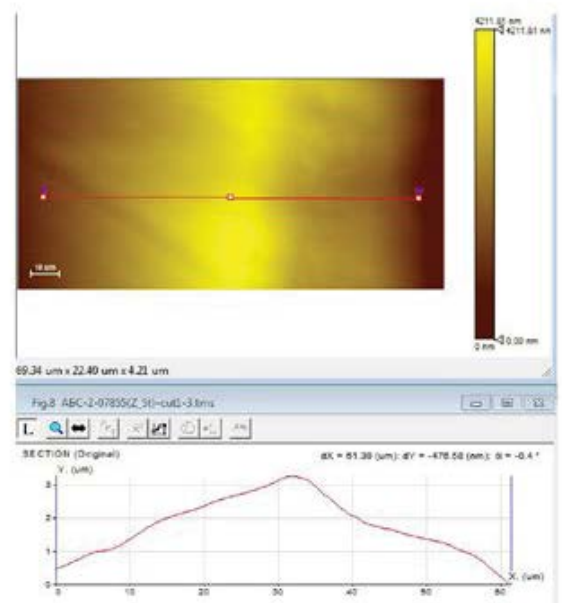

A

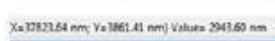

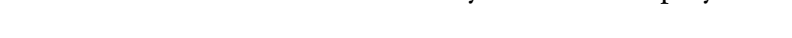


coating). Secondly, the presence of morphological defects simultaneously with a uniform distribution of mechanical characteristics of Stent 1 coating indicates that not sufficiently optimized technology of applying of the polymer composition was not used. The differences in technology of the coatings formation on the stent surface also was shown by the data of SEM and XPS. The share of uncoated areas in the case of Stent 1 was about $17 \%$, and in the case of Stent 2 and 3 only $0.1 \%$ of the total area.

SPM data enabled further determined the variations in the composition of the used polymer components, in particular, various degree of their crystallinity. Polymers in the crystalline state have greater strength, less fluidity, and they able to form highly oriented structures as compared to amorphous polymers. One would suppose the necessity to avoid the formation of hard and solid coatings on the stent surfaces, since the latter are undergone by cycles of compression/ decompression, and therefore, the coating must possess a certain elasticity to maintain integrity during a stent installation.

Thus, it can be concluded that relatively high value of the modulus of elasticity does not prevent the formation of defects in the process of polymer coating formation on the metal surface of the stent.

\section{Conclusion}

Irregularities in the polymeric coating of stents may result from incorrect selection of components in the polymer composition which determines physicalchemical and mechanical properties of the coating, from non-optimal choice of deposition conditions, as well as from stent processing after deposition. Any of these causes can lead to the formation of morphologically similar or different irregularities including cracks, wrinkles, appearance of exposed substrate areas, lapping, craters or bubbles and conversely, coating thinning, fragmentation, delamination, etc. In this regard the study of texture, morphology and chemical composition of the surface layer of polymer-coated stents, as well as assessment of changes in surface morphology and stability of the stent coating as a result of its expanding, is a very important practical task.

The present study demonstrates that none of the individual analytical methods used in this work permits in itself to simultaneously and comprehensively evaluate morphology features of surface layer of polymer coating, mechanical properties (hardness and crystallinity) of coating and change in its chemical composition through the thickness. Table 3 lists the types of irregularities determined by the methods used in this paper in accordance with the classification proposed in [8]. Table 3 demonstrates that SEM is the most informative method for obtaining the data on the presence and types of defects in the polymer coating. This method provides an identification of virtually all types of defects depending on selected resolution. Despite the fact that the SPM and XPS, from this point of view, are inferior to the information content on of the SEM method, each of them provides additional unique advantages in the study of the stent surface.

In particular, SPM not only provides the detailed images of polymer coating on the stent surface, which are especially informative in combination with the corresponding profiles of the coating thickness changing, but also allows one to infer the physical and chemical state of the polymer coating, as well as to identify a microphase separation (stratification).

From a practical point of view, the data obtained by the XPS method is of particular interest. This method allows to evaluate the thickness and uniformity of coating to determine chemical composition of the coating and its variation in thickness and get information about the occurrence of layers with different chemical composition (for example, the presence of additional adhesive layer or multilayer coatings, etc.). In addition, it allows getting information about distribution of medical preparations, radiopaque agents, and others, which are included in the coating composition. XPS method could be particularly useful for the study of biodegradation profiles of coating (or stent material) and drug release. Therefore, the morphological changes in the surface layer of coating, which can be caused

\begin{tabular}{|c|ccc|}
\hline \multicolumn{4}{|c|}{ Table 3: Types of irregularities in the polymer coating of stent, defined by different methods. } \\
\hline Method & Type of identified defects by Category \\
\hline Optical microscopy & - & C, D & III \\
\hline SEM & A, B, C & A, B, C, D, * & A, B, C, D, E \\
\hline SPM & B & A, C & A, B, C, D \\
\hline XPS & A, C & A, C, D & A \\
\hline
\end{tabular}

* -irregularities caused by influence of residual active components of the used polymer blend. 
by these processes at any step of biodegradation, also may be accompanied by unwanted and potentially dangerous effects (premature exposure of areas of the stent structure, separation of coating fragments, etc.).

It is important that the results obtained using each of the above methods, would be useful to supplement with the data from other methods that significantly increases the effectiveness of their complex application.

Therefore, a comprehensive approach to the study of defects in the polymer coating and its composition by means of instrumental analytical methods used in this study provides the comparative evaluation of different technologies for producing of stents, identification of materials quality, to obtain valuable characteristics of the polymer coating and, finally, to give preliminary science-based estimation of the stent quality.

\section{Future Work}

Future work will be devoted to detailed study of possible connection between the causes of coating irregularities that appear during the stent expanding depending on design of stent metal structure and mechanical properties of polymer coatings, in particular, to the comparison of coating adhesion strength, hardness and Young's modulus and thickness of the selected coating. It is planned to carry out mathematical modeling of the stent expanding process for the stents of different design.

\section{Executive summary}

Background: The clinical performance of a drug-eluting coronary stent largely depends on the properties of its polymeric coating. While the randomized clinical research is the "gold standard" for the effectiveness and safety evaluation of the coronary stent, the bench top testing of the stent coating may provide valuable insights within the development of new devices. We argue that the combination of the instrumental analytical methods discovers more information of the nature and possible causes of irregularities of the stent coating than scanning electron microscopy conventionally used for this purpose.

Methods and Findings: We examined bioresorbable coatings of three commercially available DES using scanning electron microscopy (SEM), scanning probe microscopy (SPM), nanoindentation, and X-ray photoelectron spectroscopy (XPS) to evaluate morphology (thickness in particular) and mechanical properties of the coating, as well as the of elemental and chemical composition through the coating thickness.

Conclusions: Adding SPM and XPS methods to SEM for analysis of DES surface provides additional value, especially for the development of new polymeric coating. For the coatings being analyzed, the SEM method revealed the difference in the uncoated surface as big as $0.1 \%$ and $17 \%$ for different models of the stents. The data obtained by XPS method confirmed the results of the SEM-study about the surface uniformity. The depth profiling of the polymer drug-containing layers also showed the difference in thickness of the coatings as well as allowed to determine elemental components of layers. The SPM method allows excluding the heterogenic or difference of elastic properties of the polymer coating as a cause for coating irregularity, that appear to differ only slightly for the stents with the smallest and largest uncoated areas.

Further research is needed to find correlation of the properties discovered with analytical methods with characteristics of biological response.

\section{References}

1. Morice MC, Serruys PW, Sousa JE, et al. A randomized comparison of a sirolimus-eluting stent with a standard stent for coronary revascularization. N. Engl. J. Med. 346: 1773-1780 (2002).

2. Moses JW, Leon MB, Popma JJ, et al. Sirolimuseluting stents versus standard stents in patients with stenosis in a native coronary artery. N. Engl. J. Med. 349: 1315-1323 (2003).

3. Stone GW, Ellis SG, Cox DA, et al. A polymerbased, paclitaxeleluting stent in patients with coronary artery disease. N. Engl. J. Med. 350: 221-231(2004).

4. Daemen J, Wenaweser P, Tsuchida K, et al. Early and late coronary stent thrombosis of sirolimus-eluting and paclitaxel-eluting stents in routine clinical practice: data from a large twoinstitutional cohort study. Lancet. 369: 667-678 (2007).

5. Jensen LO, Tilsted HH, Thayssen P, et al. Paclitaxel and sirolimus eluting stents versus bare metal stents: long-term risk of stent thrombosis and other outcomes. From the Western Denmark Heart Registry. Euro. Intervention. 5: 898-905 (2010).

6. Guidance for Industry. Coronary Drug-Eluting Stents. US Department of Health and Human Services Food and Drug Administration. (2008).

7. Glocker DA, Hoboken SV. Medical coatings and deposition technologies. John Wiley and Sons, New Jersey. pp: 978 (2016).

8. Basalus MW, Ankone MJ, van Houwelingen GK, de Man FH, von Birgelen C. Coating irregularities of durable polymer-based drug-eluting stents as assessed by scanning electron microscopy. Euro. Intervention. 5: 157-165 (2009). 